\title{
A BIOLOGICALLY INSPIRED NEURAL NETWORK COMPOSED OF DISSIMILAR SINGLE NEURON MODELS
}

\author{
P. Poirazi ${ }^{1}$, C. C. Neocleous ${ }^{2}$, C. S. Pattichis ${ }^{1}$ and C. N. Schizas ${ }^{1}$ \\ ${ }^{1}$ Department of Computer Science, University of Cyprus, 75 Kallipoleos, 1678 P.O. Box 20537, \\ Nicosia, Cyprus. Email:poirazi@LNC.usc.edu. pattichi@ucy.ac.cy, கchizas@ucy.ac.cy \\ ${ }^{2}$ Mechanical Engineering Department, Higher Technical Institute, P.O. Box 20423 \\ Nicosia, Cyprus. Email: costas@ucy.ac.cy
}

\begin{abstract}
A multilayer neural network has been developed that consists of slabs of single neuron models. Each slab is composed of a single type of neurons, which differs between the slabs. The network was trained using a biologically inspired, Hebbian-like, learning rule on EMG data and good training/testing classification performance was obtained. It was shown that the biologically inspired network, the novel architecture of which is derived from the functionally distinct hypercolumns of neurons in the brain, can be successfully applied on difficult classification tasks.
\end{abstract}

Keywords: neural networks, dissimilar neuron models, Hebbian learning rule.

\section{INTRODUCTION}

Neural tissue has the ability to learn and memorize a vast amount of information over very short time scales with little exposure to the learning data. The mechanisms underlying learning and memory processes in the brain are only partially identified to this date. Biophysical and computational studies indicate that information is learned through a process of structural modifications that take place in synaptic terminals between neurons, in response to the frequency of activation [1-3]. This process is believed to be guided by locally available signals at synaptic sites (Hebbian learning rules) while it could also be partially stochastic and partially regulated by backpropagating messages sent from the cell body to the modifiable sites [46]. Previous computational work has shown that such a mechanism could significantly boost the memory capacity of biological neurons [7-9]. In this work, a supervised Hebbian-Stochastic learning rule is implemented in a novel neural network model and the performance is compared to existing rules. The architecture of the proposed model is also inspired from the morphological and biophysical properties of neurons in the brain. Specifically, the model is composed of three layers where the neurons in the hidden layer are divided in two distinct categories (slabs). Each slab is made of identical single neuron models but neurons between the slabs are different. This differentiation in the neuronal processing properties, which in the neural network case is depicted by using different single neuron models, has been observed in the primary visual cortex (V1). In the 1960's, Hubel and Wiesel described cells in V1 which responded to the image of oriented bars and edges $[10,11]$. The most basic cell type, which they described, responded to a stationary, spatially localized oriented contour and this cell was dubbed the simple cell. Hubel and Wiesel also reported a striking regularity in the organization of the cells in V1 based upon cortical columns (or hypercolumns) running tangentially to the cortical surface. They showed that neurons selective to a continuous range of different orientations were grouped together in one hypercolumn [11, 12]. This functional differentiation has been suggested to enhance learning by promoting decorrelation of the information learned by different parts of the physical network. Based on this evidence, the present work investigates the classification performance of a novel neural network architecture, where the hidden layer of a three-layer network is divided into sets of different single neuron models.

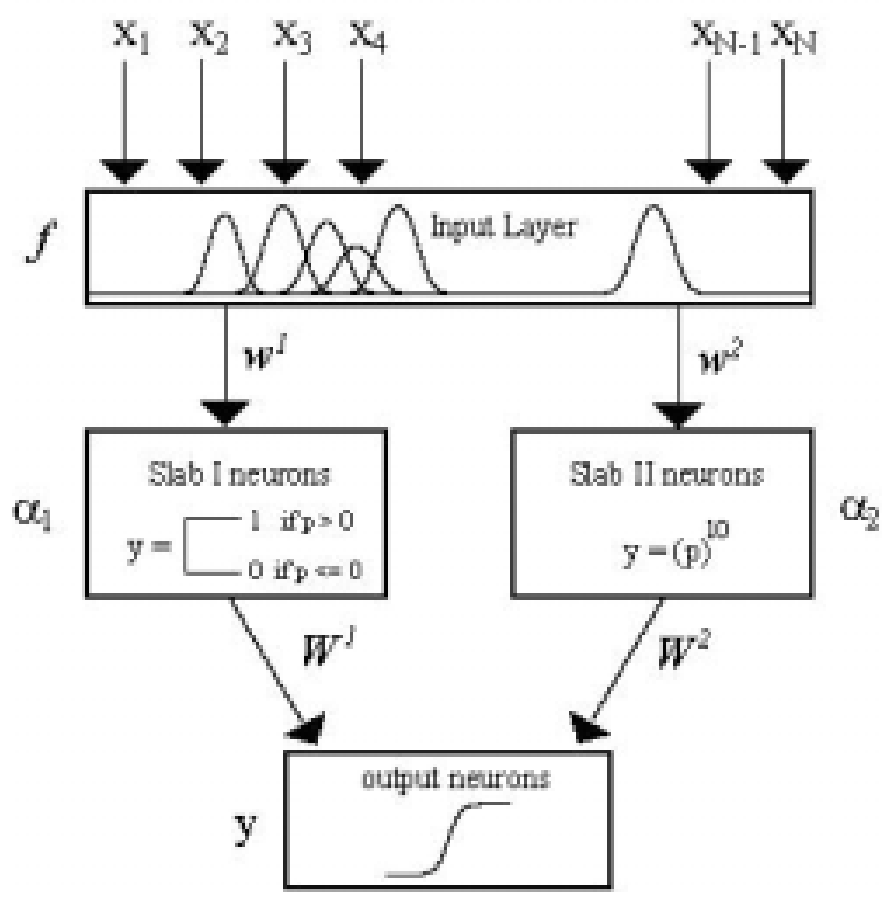

Figure 1.

The proposed neural network is composed of three layers: an input layer, two hidden slabs with dissimilar neurons and an output layer. The size of the network in the present study is 24 input neurons, 10 hidden neurons ( 5 per slab) and three output neurons. 


\section{Report Documentation Page}

\begin{tabular}{|l|c|c|}
\hline $\begin{array}{l}\text { Report Date } \\
25 \text { Oct } 2001\end{array}$ & $\begin{array}{c}\text { Report Type } \\
\text { N/A }\end{array}$ & Dates Covered (from... to) \\
\hline
\end{tabular}

\section{Title and Subtitle}

A Biologically Inspired Neural Network Composed of Dissimilar Single Neuron Models

\begin{tabular}{|l}
\hline Author(s) \\
\hline $\begin{array}{l}\text { Performing Organization Name(s) and Address(es) } \\
\text { Kallipoleos } 1678 \text { P.O. Box } 20537 \text { Nicosia, Cyprus }\end{array}$ \\
\hline $\begin{array}{l}\text { Sponsoring/Monitoring Agency Name(s) and Address(es) } \\
\text { US Army Research, Development \& Standardization Group } \\
\text { (UK) PSC 802 Box 15 FPO AE 09499-1500 }\end{array}$ \\
\hline
\end{tabular}

\section{Distribution/Availability Statement}

Approved for public release, distribution unlimited

\section{Supplementary Notes}

Papers from 23rd Annual International Conference of the IEEE Engineering in Medicine and Biology Society, October 25-28, 2001, held in Istanbul, Turkey. See also ADM001351 for entire conference on cd-rom., The original document contains color images.

\begin{tabular}{|l} 
Abstract \\
\hline Subject Terms \\
\hline $\begin{array}{l}\text { Report Classification } \\
\text { unclassified }\end{array}$ \\
\hline
\end{tabular}

Classification of Abstract

unclassified

\section{Number of Pages}

4
Contract Number

Grant Number

Program Element Number

Project Number

Task Number

Work Unit Number

Performing Organization Report Number

Sponsor/Monitor's Acronym(s)

Sponsor/Monitor's Report Number(s)
Classification of this page

unclassified

\section{Limitation of Abstract}

UU 


\section{METHODOLOGY}

Previous work has shown that a multi-slab architecture similar to the one shown in figure 1., can yield high classification scores on a variety of application [13]. Based on these earlier models, we develop a three layer feedforward neural network which consists of: (1) an input layer where input features are pre-processed with the use of adaptable Gaussian activation functions (Receptive Fields), (2) two slabs of hidden neurons where single neuron models within each slab are the same but differ between slabs and (3) an output neuron where the outputs for each slab are linearly combined before passed through an adaptable Logistic Activation Function (LAF). The Receptive Field (RF) equation for each distinct input feature $k$ is given by:

$$
f_{k}\left(x_{k}\right)=a_{k} \cdot \exp \left(-s_{k} \cdot\left(x_{k}-H_{k}\right)_{k}^{n}\right)
$$

where $x_{k}$ is the $k^{\text {th }}$ input feature of training pattern $\mathbf{x}$. Thus, the number of RFs in the input layer is equal to the number of input features in the learning set. The parameters for each RF $\left(a_{k}, s_{k}, H_{k}, n_{k}\right)$ as well as the LAF parameters $\left(B_{j}\right.$, $C_{j}$ ) shown in equation 6 are modified during learning by $\pm 10 \%$ random changes in their magnitude. Changes are only kept if they lead to lower MSE. Receptive Fields in the network are similar to spatial receptive fields of neurons in the LGN or the retina, where visual information is first processed in the brain $[10,14]$. The receptive fields of these neurons have been shown to have a Gaussian-like (center-surround) shape where stimuli that lie in the center of the receptive field (RF) excite the cell while stimuli in the periphery of the RF do not cause neuron firing. The idea behind this adaptable design is to allow each input neuron to focus on a specific subset of the input data thus promoting the decorrelation of patterns learned by different parts of the network, a method that has been shown to enhance information learning in the brain [15, 16]. The hidden layer of the network consists of dissimilar single neuron models divided in two slabs, where neurons within each slab are identical. The two types of single neuron models are given by the equations:

$$
\begin{array}{lll}
\alpha_{1}(p)=0 & \text { if } & p \leq 0 \\
\alpha_{1}(p)=1 & \text { if } & p>0
\end{array}
$$

for the first slab and

$$
\alpha_{2}(p)=p^{10}
$$

for the second slab. $p$ is the weighted sum of the postprocessed input pattern $\boldsymbol{f}=\left[f_{1}\left(\mathrm{x}_{1}\right) f_{2}\left(\mathrm{x}_{2}\right) \ldots f_{\mathrm{N}}\left(\mathrm{x}_{\mathrm{N}}\right)\right]$ :

$$
p(\boldsymbol{f})=\sum \mathrm{w}_{\mathrm{k}, \mathrm{i}}^{\mathrm{L}} \cdot f_{k}\left(\mathrm{x}_{\mathrm{k}}\right)
$$

where $\mathrm{w}_{\mathrm{k}, \mathrm{i}}{ }^{\mathrm{L}}$ is the weight between Receptive Field $k$ and neuron $i$ in slab $L=1,2$. A power of ten was selected for neurons in slab 2 based on the results of an earlier work where morphologically realistic neurons were used on classification tasks [9]. The use of dissimilar neuron models is another means of promoting the decorrelation of information learned by the two slabs of the network. The outputs of both slabs were linearly combined and fed to the third layer of the network. The activation functions for the $\mathrm{j}$ $=1, \ldots \mathrm{J}$ neurons in the output layer are given by:

$$
\mathrm{y}_{\mathrm{j}}\left(\mathbf{A}_{\mathrm{j}}\right)=B /\left(1+\exp \left(-C_{j} \cdot \mathbf{A}_{j}\right)\right)
$$

with $\mathbf{A}=\left[\begin{array}{llll}\mathbf{A}_{1} & \mathbf{A}_{2} & \ldots & \mathbf{A}_{\mathrm{N}}\end{array}\right]$ the matrix of the combined slab output:

$$
\mathbf{A}=\left[\mathrm{W}_{\mathrm{i} j \mathrm{j}}^{1} \mathrm{~W}_{\mathrm{i} \mathrm{j}}^{2}\right] \cdot\left[\alpha_{1} \alpha_{2}\right]^{\mathrm{T}}
$$

and $\mathrm{W}_{\mathrm{i} j}{ }^{1}, \mathrm{~W}_{\mathrm{i} j \mathrm{j}}{ }^{2}$ equal to the weights between the hidden layers and the output layer of the network.

Hebbian Annealing Rule (HAR): A biologically inspired learning rule was developed which is compared to a random modifications rule used in previous studies [17]. Learning in the proposed network proceeds as follows: (1) weights and activation function variables are initialized at random (2) at each step, a neuron (in each slab and output layer) is selected at random and three weights from the weight matrix associated with this neuron are randomly selected. A score is calculated for each weight as shown by the equation:

$$
S_{w}=\operatorname{cor}\left(\mathbf{M}_{\text {in }}(\boldsymbol{I}), M_{\text {out }}(\boldsymbol{I})\right)
$$

where the function $\operatorname{cor}(\boldsymbol{a}, \boldsymbol{b})$ calculates the correlation coefficient between vectors $\boldsymbol{a}$ and $\boldsymbol{b}, \mathbf{M}_{\text {in }}$ is the row of the input matrix corresponding to the selected weight $\mathrm{w}$ (or W), $\mathbf{M}_{\text {out }}$ is the corresponding row of the output matrix for the selected neuron, and $\boldsymbol{I}$ is an index used to identify all misclassified patterns. Thus, $\boldsymbol{M}_{\text {in }}(\boldsymbol{I})$ and $\boldsymbol{M}_{\text {out }}(\boldsymbol{I})$ indicate that the correlation is measured only over the patterns that were misclassified in the specific run. This method was previously shown to lead in faster convergence of the algorithm [9]. The score for each weight in the selected pool is calculated and the weight with the smaller score is targeted for modification. The modification is a $\pm 10 \%$ change in its magnitude and the change is kept or rejected based on a Boltzman equation:

$$
\mathrm{R}=1 /\left(1+\exp \left(\left(\mathrm{E} \_ \text {new-E_old }\right) / \mathrm{T}\right)\right)
$$


A change is kept if E_new $<$ E_old or a random number a $\in[0,1]$ is smaller than the result of equation (9). A temperature variable $(T)$ is lowered by a scaling factor $\left(T_{f}\right)$ over the course of learning such than fewer bad changes are accepted as the algorithm converges to a minimum. To avoid local minima, an additional criterion was incorporated in the learning rule. If the error rate was unchanged for 300 consecutive iterations, $\mathrm{T}$ was increased by a factor of $\operatorname{sqrt}\left(\mathrm{T}_{\mathrm{f}}\right)$. For the experiments reported here, the initial Temperature $\mathrm{T}=20$ and the temperature reduction factor $\mathrm{T}_{\mathrm{f}}=0.95$. Learning was terminated after a maximum number of iterations $(20,000)$ or when no further improvement in the MSE was observed after 1000 consecutive iterations.

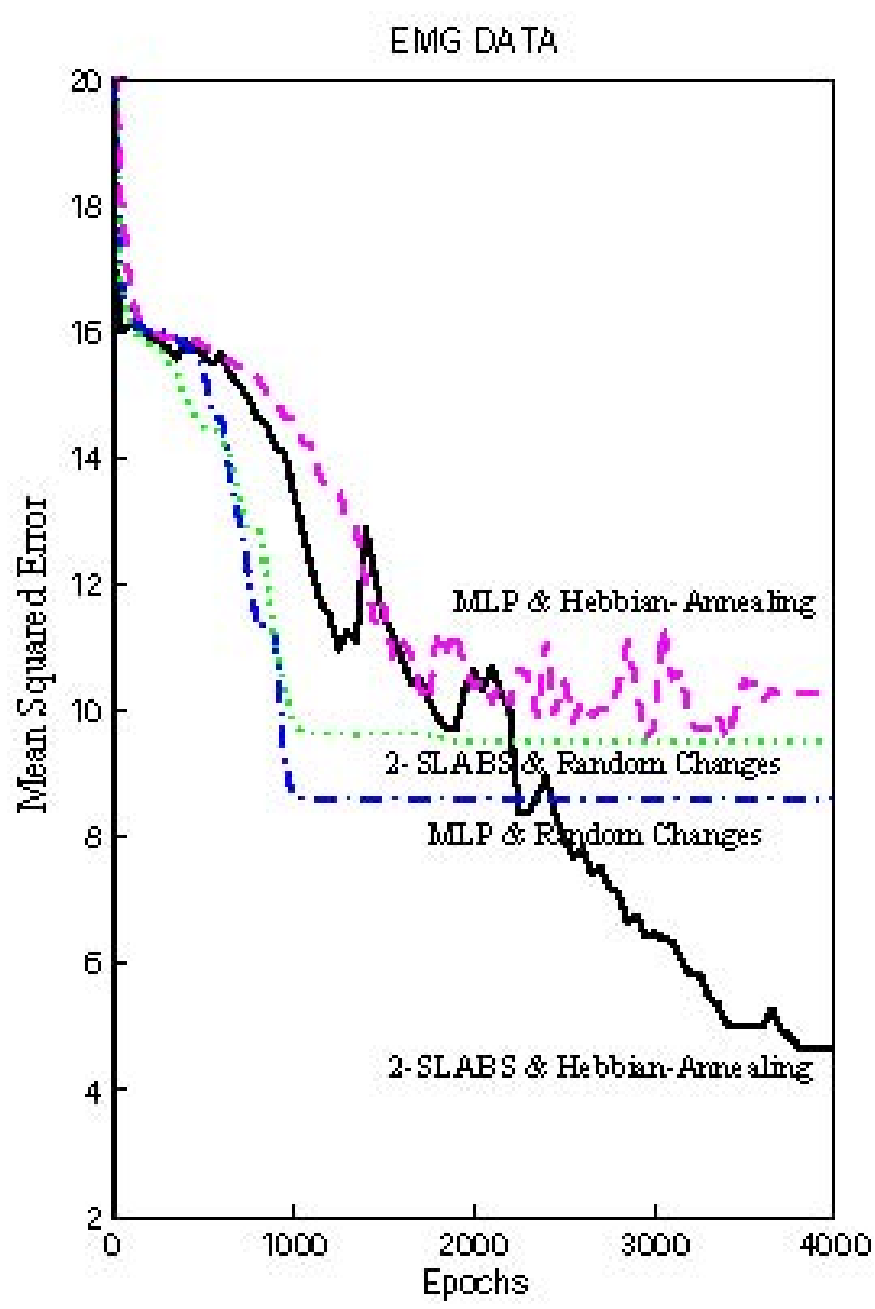

Figure 2.

Two network architectures were trained with both the Hebbian-Annealing (HA) and the Random Modification (RM) rule. The first network consisted of identical neurons in both hidden layers (dashed and dasheddotted lines: essentially linear neurons with a step activation function shown in (3)) and the second network is depicted in figure 1 (solid and dotted lines). We show that combination of HA rule and dissimilar neuron network results in significantly better performance than the RM rule. Moreover, the proposed architecture/learning rule combination has the faster convergence than all cases tested.

\section{RESULTS}

The neural network model was used for the classification of Electromyography data and its performance was compared to a learning rule with random parameter modifications (RM) versus the Hebbian Annealing (HA) rule. The $\mathrm{RM}$ rule consisted of $\pm 10 \%$ random modifications at weight, $\mathrm{RF}$ and activation function parameters, alternatively, and the changes were kept only if they resulted in lower MSE rate. The above rule was selected for comparison since it was previously shown to be superior to both backpropagation and simulated annealing rules in a neural network with similar architecture [17].

MATERIAL: Motor unit action potentials (MUAPs) recorded during routine electromyographic (EMG) examination provide important information for the assessment of neuromuscular disorders. In this study we use a neural network model to analyse MUAPs using the mean and standard deviation of seven time domain parameters: duration, spike duration, amplitude, area, spike area, number of phases and number of turns [18]. A total of 480 MUAPs obtained from 24 subjects, 8 NOR (healthy), 8 MYO (myopathy) and 8 MND (motor neuron disease), were used for training the ANN classifiers, whereas a total of 200 MUAPs, obtained from 10 subjects, 4 NOR, 3 MYO and 3 MND were used for evaluation.

Due to the rather limited amount of the input data and in order to verify the correctness of the classification results a bootstrapping procedure was used. The system was trained and evaluated using five different bootstrap sets where in each set 24 different subjects were selected at random for training and 10 different subjects for evaluation. A representative error curve for both rules is shown in figure 2. The convergence rate of the biological rule is significantly faster than the corresponding random rule as shown in the graph. Finally, in addition to the MSE minimization advantage, the classification performance of the Hebbian Annealing rule is also better. The mean percentage and the standard deviation (Std) of the correct classifications score, i.e. diagnostic yield, for the five bootstrap sets for each rule is shown in Table 1 .

Table 1.

\begin{tabular}{|c|c|c|}
\hline & $\begin{array}{c}\text { Training } \\
(\% \text { correct })\end{array}$ & $\begin{array}{c}\text { Exaluation } \\
(\% \text { correct) }\end{array}$ \\
\hline $\begin{array}{c}\text { Random Changes } \\
\text { Learning Fule }\end{array}$ & $75.23 \%+\%-10 \%$ & $605 \%+1-15 \%$ \\
\hline $\begin{array}{c}\text { Hebbiam-Armealing } \\
\text { Learing Fulle }\end{array}$ & $9329 \%+2 \%$ & $86 \%+\%-5.4 \%$ \\
\hline
\end{tabular}

Training and Evaluation results for the two learning rules tested using the model architecture shown in Figure 1. Both the Training and Evaluation performance of the network trained with the Hebbian-Annealing rule are significantly better than the corresponding Random Modifications rule. In both cases, the model was trained until no further improvement in the MSE was observed after 1000 consecutive iterations. 


\section{DISCUSSION}

A novel neural network model was developed, the architecture of which combines the integrative properties of biological neurons with a neurally inspired learning rule. The three layer neural network consisted of two parallel hidden units, each composed of a distinct single neuron model type. A similar network architecture was first implemented by [17] and its benefits have yet to be explored fully. The present work has shown that such artificial neural networks, which are supported by physiological evidence in the visual system, can be successfully used on difficult memory/recognition tasks. The proposed model was trained with a supervised Hebbian-annealing learning rule, and was found to give good results on the classification problem tested. It was shown that both the model architecture and learning rule are responsible for improved performance. The artificial neural network was found to perform significantly better that a same-sized MLP network trained with either a random change rule or the biological rule. Furthermore, the proposed network performed at it's best when trained with the biological learning rule. Thus, it can be concluded that learning mechanisms employed by the brain can be successfully used in artificial learning tasks and possibly even outperform existing algorithms.

\section{REFERENCES}

[1] T. V. Bliss, \& Lynch, M.A., "Long-term potentiation: mechanisms and properties.," in Long-term Potentiation: From Biophysics to Behavior, P. W. Landfield, \& Deadwyler, S.A, Ed. New York: Alan Liss, 1988, pp. 3-72.

[2] T. V. Bliss, Clements, M.P., et al., "Presynaptic changes associated with long-term potentiation in the dentate gyrus.," Semin. Neuroscience, vol. 2, pp. 345-354, 1990.

[3] D. O. Hebb, The organization of behavior: a neurophysiological theory. New York: Wiley Press, 1949.

[4] G. Stuart, \& Sakmann, B., "Active propagation of somatic action potentials into neocortical pyramidal cell dendrit," Nature, vol. 367, pp. 69-72, 1994.

[5] N. Spruston, Schiller, Y., Stuart, G., \& Sakmann, B., "Activity-dependent action potential invasion and calcium influx into hippocampal CA1 dendrites," Science, vol. 286, pp. 297-300, 1995.

[6] G. Stuart, \& Spruston, N., "Determinants of voltage attenuation in neocortical pyramidal neuron dendrites," Journal of Neuroscience, vol. 18, pp. 3501-3510, 1998.

[7] P. Poirazi, \& Mel, B.W., "Towards the memory capacity of neurons with active dendrites," Neurocomputing, vol. 1, pp. 237-345, 1999.

[8] P. Poirazi, \& Mel, B. W., "Choice and value flexibility jointly contribute to the capacity of a subsampled quadratic classifier," Neural Comput, vol. 12, pp. 1189-205., 2000.

[9] P. Poirazi, \& Mel, B.W., "Impact of active dendrites and structural plasticity on the memory capacity of neural tissue," Neuron, vol. 29, pp. 779-96., 2001.

[10] D. H. Hubel, \& Wiesel, T.N., "Receptive fields, binocular interaction and functional architecture in the cat's visual cortex," Journal of Physiology, London, vol. 160, pp. 106154, 1962.

[11] D. H. Hubel, \& Wiesel, T.N., "Functional architecture of the macaque monkey visual cortex.," Proceedings of the Royal Society, London [B], vol. 198, pp. 1-59, 1977.

[12] R. Vogels, \& Orban, G.A., "How well do the response changes of striate neurons signal differences in orientation: A study in the discriminating monkey.," Journal of Neuroscience, vol. 10, pp. 3543--3558, 1990.

[13] C. Neocleous, \& Schizas, C.N., "A Neural Network Architecture Composed of Adaptively Defined Dissimilar Single-Neurons: Applications in Engineering and Design.,". U.K.: Brunel University, 1997.

[14] D. Daqing C., G.C., \& Freeman, R.D., "Spatiotemporal Receptive Field Organization in the Lateral Geniculate Nucleus of Cats and Kittens," Journal of Neurophysiology, vol. 78, pp. 1045-1061, 1997.

[15] G. Indiveri, Raffo, L., Sabatini, S.P., \& Bisio, G.M, "A neuromorphic architecture for cortical multi-layer integration of early visual tasks," Machine Vision and Applications, vol. 10, pp. 305-314, 1995.

[16] G. Indiveri, Raffo, L., Sabatini, S.P., \& Bisio, G.M, "A recurrent neural architecture mimicking cortical preattentive vision systems.," Neurocomputing, vol. 11, pp. 155-170, 1996.

[17] C. Neocleous, Esat, I.I., \& Schizas, C.N., "A Neural Network Architecture Composed of Adaptively Defined Dissimilar Single-Neuron Units," presented at Proceedings of the 2nd International Conference on Computational Intelligence and Neuroscience, Druham, 1997.

[18] C. S. Pattichis, Schizas, C.N., Middleton, L.T., "Neural Network Models in EMG diagnostics," IEEE Transactions on Biomedical Engineering, vol. a42, pp. 486-496, 1995. 